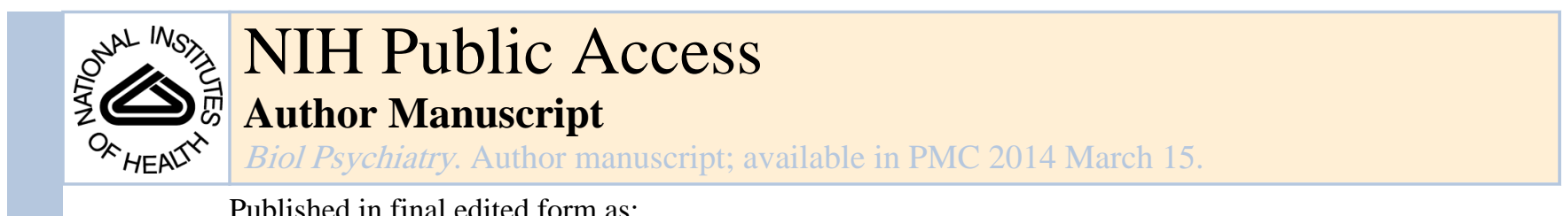

Biol Psychiatry. 2013 March 15; 73(6): 565-573. doi:10.1016/j.biopsych.2012.07.031.

\title{
Global Prefrontal and Fronto-amygdala Dysconnectivity in Bipolar I Disorder with Psychosis History
}

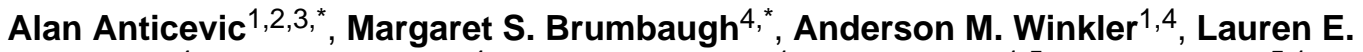 \\ Lombardo $^{4}$, Jennifer Barrett ${ }^{4}$, Phillip R. Corlett ${ }^{1}$, Hedy Kober ${ }^{1,5}$, June Gruber ${ }^{5,1}$, Grega \\ Repovs $^{6}$, Michael W. Cole ${ }^{7}$, John H. Krystal ${ }^{1,2,3}$, Godfrey D. Pearlson ${ }^{1,4,8}$, and David C. \\ Glahn ${ }^{1,4}$ \\ ${ }^{1}$ Department of Psychiatry, Yale University School of Medicine, 300 George Street, New Haven, \\ CT, 06511, USA \\ ${ }^{2}$ NIAAA Center for the Translational Neuroscience of Alcoholism, New Haven, CT 06519 \\ ${ }^{3}$ Abraham Ribicoff Research Facilities, Connecticut Mental Health Center, New Haven, CT
}

(C) 2012 Society of Biological Psychiatry. Published by Elsevier Inc. All rights reserved.

Corresponding Author: Alan Anticevic Ph.D., Yale University, Department of Psychiatry, 34 Park St., New Haven, CT 06519, Office (203) 974-7763, alan.anticevic @yale.edu.

These authors made equal contributions to the manuscript.

Financial Disclosures

Dr. John H. Krystal discloses the following: Consultant Note: - The Individual Consultant Agreements listed below are less than $\$ 10,000$ per year

Aisling Capital, LLC; AstraZeneca Pharmaceuticals; Biocortech; Brintnall \& Nicolini, Inc.; Easton Associates; Gilead Sciences, Inc.; GlaxoSmithKline; Janssen Pharmaceuticals; Lundbeck Research USA; Medivation, Inc.; Merz Pharmaceuticals; MK Medical Communications; F. Hoffmann-La Roche Ltd; SK Holdings Co., Ltd; Sunovion Pharmaceuticals, Inc.; Takeda Industries; Teva Pharmaceutical Industries, Ltd.

Scientific Advisory Board

Abbott Laboratories; Bristol-Myers Squibb; Eisai, Inc.; Eli Lilly and Co.; Forest Laboratories, Inc.; Lohocla; Research Corporation;

Mnemosyne Pharmaceuticals, Inc.; Naurex, Inc.; Pfizer Pharmaceuticals; Shire Pharmaceuticals

Exercisable Warrant Options

Tetragenex Pharmaceuticals (value less than \$150)

Board of Directors:

Coalition for Translational Research in Alcohol and Substance Use Disorders

President Elect:

American College of Neuropsychopharmacology

Research Support to Department of Veterans Affairs

Janssen Research Foundation (Provided drug and some study support to the Department of Veterans Affairs)

Editorial Board

Income Greater than $\$ \mathbf{1 0 , 0 0 0}$

Editor - Biological Psychiatry

Employment:

Yale University School of Medicine

VA CT Healthcare System

Patents and Inventions

1) Seibyl JP, Krystal JH, Charney DS. Dopamine and noradrenergic reuptake inhibitors in treatment of schizophrenia. Patent \#: 5,447,948. September 5, 1995

2) I am a co-inventor with Dr. Gerard Sanacora on a filed patent application by Yale University related to targeting the glutamatergic system for the treatment of neuropsychiatric disorders (PCTWO06108055A1).

3) Intranasal Administration of Ketamine to Treat Depression (pending)

Dr. Corlett reports no biomedical financial interests or potential conflicts of interest relevant to this work, but for unrelated activities, consults for Pfizer and Johnson \& Johnson and has received research support from Pfizer and AstraZeneca. All other authors report no biomedical financial interests or potential conflicts of interest.

Publisher's Disclaimer: This is a PDF file of an unedited manuscript that has been accepted for publication. As a service to our customers we are providing this early version of the manuscript. The manuscript will undergo copyediting, typesetting, and review of the resulting proof before it is published in its final citable form. Please note that during the production process errors may be discovered which could affect the content, and all legal disclaimers that apply to the journal pertain. 
${ }^{4}$ Olin Neuropsychiatry Research Center, Institute of Living, Hartford Hospital, 200 Retreat Avenue, CT, 06106, USA

${ }^{5}$ Department of Psychology, Yale University, 2 Hillhouse Avenue, P.O. Box 208205, New Haven, CT, 06520, USA

${ }^{6}$ Department of Psychology, University of Ljubljana, Ljubljana, Slovenia

${ }^{7}$ Washington University in St. Louis, St. Louis, MO, 63130

${ }^{8}$ Department of Neurobiology, Yale University, 333 Cedar St., New Haven, CT, 06510, USA

\section{Abstract}

Background-Pathophysiological models of bipolar disorder postulate that mood dysregulation arises from fronto-limbic dysfunction, marked by reduced prefrontal cortex (PFC) inhibitory control. This may occur both due to disruptions within PFC networks and abnormal inhibition over subcortical structures involved in emotional processing. However, no study has examined global PFC dysconnectivity in bipolar disorder and tested if regions with within-PFC dysconnectivity also exhibit fronto-limbic connectivity deficits. Further, no study has investigated whether such connectivity disruptions differ for bipolar patients with psychosis history, who may exhibit a more severe clinical course.

Methods-We collected resting-state fMRI at 3T in 68 remitted bipolar I patients (34 with psychosis history) and 51 demographically-matched healthy participants. We employed a recently developed Global Brain Connectivity method, restricted to PFC (rGBC). We also independently tested connectivity between anatomically-defined amygdala and PFC.

Results-Bipolar patients exhibited reduced medial PFC (mPFC) rGBC, increased amygdalaMPFC connectivity, and reduced connectivity between amygdala and dorso-lateral PFC. All effects were driven by psychosis history. Moreover, the magnitude of observed effects was significantly associated with lifetime psychotic symptom severity.

Conclusions-This convergence between rGBC, seed-based amygdala findings and symptom severity analyses highlights that mPFC, a core emotion regulation region, exhibits both withinPFC dysconnectivity and connectivity abnormalities with limbic structures in bipolar illness. Furthermore, lateral PFC dysconnectivity in patients with psychosis history converges with published work in schizophrenia, indicating possible shared risk factors. Observed dysconnectivity in remitted patients suggests a bipolar trait characteristic and may constitute a risk factor for phasic features of the disorder.

\section{Keywords}

bipolar disorder; prefrontal cortex; amygdala; connectivity; resting-state; psychosis

\section{Introduction}

Bipolar disorder is characterized by prominent mood dysregulation(1). Pathophysiological models of bipolar illness suggest this dysregulation may arise from both dysfunction in prefrontal cortical (PFC) networks linked to cognitive control of emotion, and disruptions in prefrontal control over subcortical regions involved in affective processing like the amygdaloid complex(2). Functional magnetic resonance imaging (fMRI) findings support this model by demonstrating abnormalities across subcortical/limbic and cortical structures, notably the amygdala and medial PFC (mPFC)(3). These regions show mood-statedependent activity alterations in bipolar disorder and have been linked to emotion generation and appraisal(4-7). Moreover, individuals with bipolar disorder show aberrant prefrontal activation across cognitive challenges $(6,8)$, suggesting possible disturbances in prefrontal 
function. However, PFC is large and heterogeneous with widespread connectivity and it is unclear which specific prefrontal circuits may be compromised in this disorder. While evidence supports that localized structure and function of $\mathrm{mPFC}$ is disrupted in bipolar disorder(8), there is relatively little information about the relationships between prefrontal cortical regions in bipolar illness. Complex neuropsychiatric disease like bipolar disorder may result from disrupted neural computations across networks of regions(9). Indeed, severe mood disorders are associated with abnormal structural plasticity and cellular resilience(1012), which may give rise to impairments in distributed neural networks(9). Therefore, it is critical to identify prefrontal circuitry exhibiting distributed PFC functional abnormalities, which may relate to deficits in both PFC function and control over limbic structures. Yet, prefrontal dysconnectivity has not been systematically investigated in this illness.

A growing body of evidence shows that distributed neural circuits exhibit spontaneous activity at rest(13). These slow-frequency fluctuations are temporally correlated within spatially-distinct but functionally-related networks(14), establishing an intrinsic functional network architecture (15) across primate species(16). These networks show high concordance with other measures of structural and functional connectivity in healthy populations(17) and provide an opportunity to characterize distributed circuit abnormalities in neuropsychiatric illnesses(18). Prior research using resting-state techniques demonstrates that individuals with bipolar disorder show reduced connectivity within the "default mode network"(19), the pregenual anterior cingulate, thalamus and amygdala(20), as well as in the ventral prefrontal-amygdala pathways(21). Although these findings constitute important advances in our understanding of bipolar disorder, no study to date has investigated global prefrontal dysconnectivity patterns (i.e. across all prefrontal gray matter voxels). Such a global, data-driven approach is vital as it allows comprehensive examination of prefrontal connectivity abnormalities. This in turn offers the potential to identify specific prefrontal nodes compromised in bipolar illness, which may also relate to regulation of limbic circuits.

Although identifying global prefrontal network disruption in bipolar illness is critical, such findings do not imply fronto-limbic dysconnectivity. To establish fronto-limbic dysconnectivity, both prefrontal and limbic connectivity must be assessed in the same subjects. It is well recognized that amygdala shares dense connectivity with prefrontal cortex, most notably caudal orbitofrontal cortex, $\mathrm{mPFC}$ and anterior cingulate gyrus(22-25) - all regions implicated in regulation of emotion (among other functions). The critical point of such analyses is to independently test if the same (or similar) regions identified via global connectivity may also exhibit connectivity disturbances with the amygdala. That is, examining deficits in limbic connectivity with broad PFC circuits is key to fully characterize deficits in fronto-limbic dysregulation in bipolar disorder.

While we discussed bipolar disorder as a diagnostic category, bipolar illness is highly heterogeneous in terms of onset, symptom severity, co-morbidity, clinical course, and outcome. Such diversity implies that distinct, yet partially overlapping neurobiological mechanisms may be involved in patients with differing clinical presentations. Capitalizing on a dimensional approach(26) we can identify subpopulations of patients with common symptoms or illness-course who may exhibit shared neural dysfunction. One potential axis upon which to subdivide bipolar disorder is the presence or absence of psychotic symptoms. Psychotic symptoms are present in 50-70\% of individuals with bipolar disorder $(27,28)$ and psychosis aggregates within families of bipolar patients(29). Lifetime history of psychosis may represent a more severe form of the illness associated with poorer prognosis((30, 31); but see(32)), cognitive performance(33), brain structure(34) and function(35). Recent reports of global prefrontal dysconnectivity in schizophrenia(36) raises the intriguing hypothesis that history of psychosis in bipolar disorder may be associated with more severe 
patterns of prefrontal dysconnectivity. However, prefrontal dysconnectivity has yet to be examined in psychotic bipolar disorder.

Our goal was to investigate prefrontal-limbic dysconnectivity in bipolar disorder. We tested three hypotheses: First, we examined whether there are global PFC connectivity abnormalities in this illness by applying a recently developed global brain connectivity method(37-39), which may particularly manifest in mPFC. Second, we compared patients with a history of psychosis versus patients without psychosis to determine if psychotic patients exhibit more severe PFC dysconnectivity, similar to findings in schizophrenia(36). Third, we examined functional connectivity between the amygdala and PFC using independent anatomically-delineated seeds. We specifically tested whether regions showing global prefrontal disturbances exhibit convergence with amygdala dysconnectivity.

\section{Methods}

\section{Participants}

Participants provided informed consent approved by the IRB at Hartford Hospital and Yale University. Sixty-eight remitted patients with bipolar I disorder and 51 demographicallymatched healthy individuals participated in the study (Table 1). Patients were identified through outpatient clinics and community mental health facilities in the Hartford area. Inclusion criteria for patients were: i) bipolar I disorder diagnosis as determined by the Structured Clinical Interview (SCID) for the Diagnostic and Statistical Manual of Mental Disorders-IV (DSM-IV)(40), administered by experienced MA or PhD-level research clinicians; ii) no history of major medical or neurological conditions (e.g. epilepsy, migraine, head trauma with loss of consciousness); and iii) IQ>80 assessed by Wechsler Abbreviated Intelligence Scale(41). To increase ecological validity of the patient sample, comorbid Axis I anxiety disorders and/or history of substance abuse (fully remitted $>6$ months prior to the study) were allowed. Healthy participants were recruited through media advertisements and flyers posted in the Medical Center area. Inclusion criteria for healthy participants were: i) no current or lifetime Axis I psychiatric disorder as assessed by SCIDNP; ii) no history of medical or neurological conditions; and iii) no history of psychotic or mood disorders in first-degree relatives (reported by detailed family history). While groups were matched for age, ethnicity, and sex, healthy participants' education attainment was greater than that of patients with bipolar disorder $(\mathrm{p}=.01)(42)$. Education differences are impacted by the illness course(43) and thus were not included as a covariate; alcohol, drug use, anxiety, age, illness duration, gender and medication type did not alter reported effects.

\section{Current Symptoms \& Medication}

Severity of current mood symptoms was determined with the 21-item Hamilton Depression scale (HAM-D)(44), the Young Mania Rating Scale (YMRS)(45), and the expanded version of the Brief Psychiatric Rating Scale (BPRS)(46). Only remitted patients were included in the current experiment ( $>1$ week), defined using standardized cutoffs on the HAM-D (less than or equal to 7) and YMRS (less than or equal to 7) (Table 1). 53\% of bipolar patients were receiving mood stabilizers, $43 \%$ were taking antidepressants, 34\% were taking atypical antipsychotics, $35 \%$ were taking anxiolytics, $16 \%$ were on lithium, and $16 \%$ were unmedicated at the time of assessment (note: some patients were on multiple medications). As noted, reported effects were not altered when we co-varied for medication. For details on psychosis history evaluation for bipolar patients with psychosis (BPP) vs. those without psychosis (BWP) see Supplement. 


\section{GBC Analysis}

Complete fMRI acquisition and preprocessing details are presented in the Supplement. The global brain connectivity $(\mathrm{GBC})$ approach $(36,38)$ was applied using in-house Matlab tools. GBC estimates the connectivity between each individual voxel and every other voxel in the brain. In contrast, restricted GBC estimates connectivity at every voxel with every other voxel in a restricted space (referred to hereafter as restricted global brain connectivity rGBC). Here we conducted rGBC analysis restricted to voxels within subject-specific Freesurfer-based(47) prefrontal gray matter masks (see Supplement for FreeSurfer segmentations that comprised the mask). To account for between-subject differences in anatomy, before the analysis, BOLD signal within the subject-specific cortical mask was spatially smoothed with a $6 \mathrm{~mm}$ full-width-at-half-maximum (FWHM) Gaussian kernel and dilated by two voxels $(6 \mathrm{~mm})$ to account for individual differences in anatomy. Following our prior work(36), the rGBC analysis involved, for each PFC voxel, computing a correlation with every other PFC voxel, transforming the correlations to Fisher z-values, and computing the mean. This yielded a map for each subject where each voxel value represents the mean connectivity of that voxel with the rest of PFC.

\section{Amygdala Seed-based Functional Connectivity (fcMRI) Analysis}

The seed-based amygdala fcMRI closely followed our prior work(48). As with rGBC, we employed in-house Matlab tools $(49,50)$ to examine the relationship between amygdala and all PFC voxels. To this end, we computed a seed-based amygdala correlation map by extracting average time-series across all voxels in each subject's bilateral amygdala (anatomically defined through Freesurfer-based segmentation(47, 51)), which was then correlated with each PFC voxel. Next, as with rGBC, we computed a Fisher r-to-Z transform, which yielded a map for each subject where each PFC voxel value represents connectivity with the amygdala.

\section{$2^{\text {nd }}$-Level Group Analysis}

Before computing group-level statistics, individual amygdala fcMRI and rGBC correlation maps were converted to Fisher- $Z$ maps. To examine hypothesized between-group differences, these maps were entered into 1-way ANOVAs with three across-group levels (controls, BPP, BPW). Both analyses were corrected within the anatomically-defined PFC mask (95\% overlap across all subjects). Type I error correction was based on peak and cluster extent(52) ascertained via AFNI's AlphaSim with exact smoothness estimates computed from the general linear model residuals ( $\mathrm{p}<.001, \mathrm{k}=14$ voxels for $\mathrm{rGBC}$ and $\mathrm{k}=13$ for amygdala fcMRI). Results were visualized using Caret 5.5 software (http:// brainvis.wustl.edu/wiki/index.php/Caret).

\section{Results}

\section{Global Prefrontal Connectivity in Bipolar Disorder}

To test hypothesized between-group difference in $\mathrm{rGBC}$ we computed a 1-way ANOVA. Results revealed a significant Group effect centered on $\mathrm{mPFC}(\mathrm{x}=3, \mathrm{y}=32, \mathrm{z}=1)$ (Figure 1A). This effect was largely driven by reduced connectivity for bipolar patients with psychosis history. Notably, healthy participants did not exhibit any regions of reduced prefrontal connectivity relative to the bipolar group, despite virtually identical SNR. To confirm that a history of psychosis is associated with more severe prefrontal dysconnectivity, we computed two follow-up independent-sample t-tests. Patients with psychosis history showed lower mPFC rGBC compared to healthy controls $[\mathrm{t}(83)=4.31, \mathrm{p}<.001]$, and when compared to bipolar patients without psychosis history $[\mathrm{t}(66)=3.51, \mathrm{p}<.001]$ (Figure 1B). Pair-wise comparisons were also significant when corrected within the PFC mask as a whole, 
illustrating the robustness of this effect. We also present a direct comparison of controls vs. the entire sample of bipolar patients for qualitative inspection in the Supplement (Figure S1).

\section{Amygdala-Prefrontal Connectivity in Bipolar Disorder}

To circumvent region selection bias and to ensure complete independence from observed rGBC effects (see Supplement for more detailed independence considerations), we computed a separate anatomically defined amygdala seed-based analysis with PFC, and examined the main effect of Group in a 1-way ANOVA. If we were to seed from the mPFC and indeed identify differences centered around the amygdala one could raise the issue of circularity (as those functional voxels were defined using the present analysis)(53). Results revealed two foci showing significant between-group effects (Figure 2A-B): centered on $\operatorname{mPFC}(x=1, y=41, z=-3)$ and right dorso-lateral PFC (DLPFC) $(x=34, y=43, z=30)$. Again, amygdala-mPFC findings were predominantly driven by bipolar patients with psychosis history. However, in contrast to rGBC effects, patients with psychosis history showed focal increased connectivity between the amygdala and $\mathrm{mPFC}$ relative to healthy controls $[\mathrm{t}(83)=4.5, \mathrm{p}<.001]$ and relative to bipolar patients without psychosis [ $\mathrm{t}(66)=2.76, \mathrm{p}<.007]$ (Figure 2A). Conversely, for the amygdala-DLPFC region, bipolar patients with psychosis history evidenced more negative connectivity relative to controls $[\mathrm{t}(83)=4.62, \mathrm{p}<.001]$ and patients without psychosis history $[\mathrm{t}(66)=4.11, \mathrm{p}<.001]$ (Figure 2B). To allow complete interpretation of amygdala findings we present threshold-free patterns for controls and bipolar patients in the Supplement (Figure S2).

\section{Testing for Convergence of rGBC and Amygdala Connectivity Effects}

Given our questions regarding both frontal and limbic dysconnectivity, we tested whether the voxels identified through a given analysis showed convergent effects with the other analysis. That is, given complete independence of identified regions, we tested rGBC effects in the $\mathrm{mPFC}$ voxels identified via amygdala connectivity and vice versa (i.e. amygdala connectivity effects in the mPFC voxels identified via rGBC). The purpose of the convergence analysis was to test whether identified voxels across the two approaches represent functionally distinct regions. Both effects converged: i) the rGBC effect remained significant and consistent in the $\mathrm{mPFC}$ region identified via amygdala connectivity $[F(2,116)=6.8, p<.002]$; ii) the amygdala-mPFC effect remained significant and consistent in the $\mathrm{mPFC}$ region identified via $\mathrm{rGBC}$ analysis $[\mathrm{F}(2,116)=3, \mathrm{p}=.05]$. Together, these findings further argue that functionally similar effects were present for both analyses across independently identified mPFC voxels.

\section{Lifetime Psychotic Symptom Severity}

To additionally examine the association between observed dysconnectivity and psychosis, we correlated measures of lifetime psychotic symptoms derived using the Lifetime Dimensions of Psychosis Scale (LDPS) (see Supplement) with regions that revealed between-group effects. We computed a Spearman's correlation coefficient due to nonnormally distributed symptom scores (i.e. some patients had no psychotic symptoms). We focused on positive symptoms because few patients reported lifetime negative/ disorganization symptoms. There was an inverse relationship between severity of lifetime positive symptoms and $\mathrm{mPFC}$ rGBC $[\mathrm{rho}=-.22, \mathrm{p}=.07$, trend] indicating that patients with more severe lifetime positive psychotic symptoms (e.g. hallucinations and delusions) exhibit even lower mPFC rGBC (Figure 3A). In contrast, elevated amygdala-mPFC coupling was associated with increased lifetime psychotic symptom severity [rho=.31, $\mathrm{p}<.015]$ (Figure 3B), whereas lower amygdala-DLPFC coupling was associated with more severe symptoms $[$ rho=-.44, $\mathrm{p}<.0001]$ (Figure 3C). We carried out further sub-group analysis with only those 
patients exhibiting psychotic symptoms ( $\mathrm{N}=50$, see Supplement), which revealed a consistent, but attenuated pattern.

\section{Discussion}

We investigated PFC connectivity in bipolar I disorder and found, consistent with predictions: i) significant between-group differences in $\mathrm{mPFC}$ rGBC, particularly prominent for patients with psychosis history compared to those without and controls; ii) increased connectivity for amygdala-mPFC and lower connectivity for amygdala-DLPFC networks in bipolar patients relative to controls that was exaggerated in patients with psychosis history; and iii) that the magnitude of observed effects scaled with lifetime symptom severity. These findings provide evidence for distributed dysconnectivity between $\mathrm{mPFC}$ and other prefrontal regions and focal fronto-limbic dysconnectivity between mPFC and amygdala.

\section{Global Prefrontal and Fronto-Limbic Connectivity}

We hypothesized that bipolar patients would exhibit global prefrontal dysconnectivity in regions associated with affect regulation, such as $\mathrm{mPFC}$, based on prior work indicating their critical role in regulating emotion(24). Consistent with predictions, we identified a focal $\mathrm{mPFC}$ region for which patients showed reduced connectivity relative to healthy controls. These regional findings are highly consistent with both meta-analytic and seedbased neuroimaging studies reporting focal differences in bipolar disorder $(6,54)$. However, this is the first investigation to directly document reduced functional integration between $\mathrm{mPFC}$ with the rest of prefrontal cortex in bipolar disorder. Present findings illustrate that widespread prefrontal functional disruptions with $\mathrm{MPFC}$ may underlie risk for affect deregulation, which constitutes the hallmark symptom of this illness (as patients were euthymic at the time of the scan and therefore observed differences cannot be attributed to present affect regulation deficits). Interestingly, this region showed reduced global prefrontal connectivity in patients with psychosis history relative to other groups (discussed below).

As noted, $\mathrm{mPFC}$ is involved in regulation of affect through dense and reciprocal connectivity with subcortical regions implicated in generation of affective states (e.g. amygdala)(22). Yet, the rGBC analysis does not guarantee that identified regions exhibit deficits in regulation of limbic circuits. That is, the rGBC analysis included prefrontal cortex, not subcortical limbic regions, leaving open the possibility that the mPFC region identified as showing lower prefrontal connectivity might independently show reduced subcortical limbic connectivity. Thus, we examined potential convergence between rGBC and seed-based amygdala-PFC connectivity. Our independent amygdala analysis revealed a region in close proximity to the rGBC effect (although not precisely overlapping) indicating that similar cortical territories that exhibit reduced PFC integration may also be involved in reduced limbic regulation in bipolar illness. Moreover, when we tested for convergence of effects across analysis (given their statistical independence), we found highly similar results across both identified regions. Present findings further solidify through two independent but convergent approaches - that mPFC plays a critical role in the pathophysiology of bipolar illness.

Previous resting-state studies in humans and tracing studies in primates have shown that a portion of the medial PFC exhibits positive connectivity with the amygdala(22, 24, 25, 5558 ), which we observed in our prior work(48) and here (threshold-free amygdala maps shown in Supplement). In Figure 2A the identified mPFC region exhibits low connectivity with amygdala in controls, which is increased in patients. Therefore, what does it mean if connectivity exists in a patient population that is 'low' in healthy subjects? It has been wellestablished that functional connectivity is dynamic and state-dependent(59) whereby a low 
resting-state value may change and become more positive during times when emotional regulation is warranted. Thus, the observed increased values in bipolar illness may reflect a 'state' that exists due to a heightened need for mood regulation (as proposed in the context of fear extinction,(60)). There is also the possibility that the connection 'weight' changes from frequent attempts to regulate mood. In other words, patients may be in a different state in day-to-day life frequently enough that resting amygdala-mPFC connectivity has altered (in a Hebbian sense)(59). We acknowledge that these hypotheses are speculative, yet they highlight scenarios where low coupling in the normative sample, but an increase in the clinical sample, may reflect a meaningful disturbance in amygdala-mPFC connectivity. Further work is needed to verify these possibilities. In addition, we opted for a PFC-wide amygdala seed-based analysis (as opposed to a restricted one) to verify whether amygdala seed-based results converge with those identified via rGBC (which may occur in places outside of functionally restricted patterns). Therefore, future studies should additionally constrain analyses to the medial PFC showing significant connectivity with the amygdala in healthy subjects (to add further power).

Lastly, given present focal findings, one direction that may further elucidate the pathophysiology of bipolar illness, is to relate observed dysconnectivity patterns that are predictive of symptoms with spatial patterns of gene expression known to affect cortical development(61). Recent advances in transcriptomics offer a quantitative approach toward characterizing the transcriptional landscape of PFC. Relating these spatial gene expression maps to fMRI offers ways to constrain our search for genes that exhibit expression in areas showing functional abnormalities with our neuroimaging markers. We acknowledge that bipolar illness is not exclusively genetic, but rather that indexes of dysconnectivity derived using novel measures could be employed to track spatio-temporal expression of genes that confer risk for development of bipolar illness.

\section{Prefrontal Dysconnectivity and Psychosis}

We examined the association between psychotic symptoms and prefrontal dysconnectivity in three ways: i) comparison of psychotic bipolar patients with controls; ii) comparison of patients with and without a history of psychosis; and iii) examination of lifetime history of psychosis severity and prefrontal dysconnectivity. All three comparisons indicated that psychotic bipolar patients exhibit a more severe pattern of $\mathrm{mPFC}$ rGBC and amygdala$\mathrm{mPFC} / \mathrm{DLPFC}$ coupling, further highlighted by individual-difference analyses. Interestingly, there was a mirror-like pattern between $\mathrm{mPFC}$ rGBC and amygdala-mPFC coupling, possibly reflecting reduced within-PFC integration, but higher connectivity due to compensatory regulation over the amygdala (previously reported for mPFC-insula coupling(54)). Importantly, given that patients were asymptomatic at the time of assessment, our findings support the notion that observed dysconnectivity constitutes a trait-like feature and may be related to illness risk and relapse vulnerability rather than current psychotic symptom expression. Thus, mPFC dysconnectivity may be a marker for disease risk, a possibility worth examining in at-risk or prodromal populations.

These results also extend prior findings of reduced prefrontal connectivity in schizophrenia, which were centered on right DLPFC and left inferior frontal junction(36). Although present rGBC analysis in bipolar illness only identified mPFC dysconnectivity, we found reduced amygdala-DLPFC connectivity in bipolar disorder that was particularly associated with psychosis. In contrast, higher amygdala-mPFC connectivity was present even in patients without psychosis history. One possibility is that while mPFC dysconnectivity may constitute a risk factor for bipolar disorder more generally, lateral prefrontal dysfunction may be particularly associated with risk for psychotic symptoms. Thus, present results suggest a two-part hypothesis whereby different aspects of frontal dysconnectivity may be responsible for psychosis vs. mood instability $(62,63)$. One possibility is that psychosis and 
mood instability may arise due to separate processes that overlap in their anatomy and may be inherited together through distinct vulnerabilities combining via mechanisms such as assortative mating to yield psychotic bipolar illness. An alternative possibility is that these apparently separate clinical illnesses represent different phenomenological expressions of the same underlying problem at a neural circuit level, consistent with the proposal suggested by the Research Domain Criteria (RDoC) initiative(26).

Future studies should further delineate common and unique aspects of neuropathology underlying these co-morbid but distinct symptom presentations. Current findings illustrate the need for a direct comparison of clinical groups presenting with psychotic symptoms, but possibly uniquely different aspects of cortical neuropathology. A complicating factor between investigations is psychotic illness duration/severity and its effect on prefrontal cortical circuits. It is possible that illness duration differentially impacts patterns of cortical connectivity. Similarly, acute psychotic states may be marked by a distinct pattern of prefrontal connectivity disruptions than those found in chronic patients(64). Thus, future work should quantify differences in prefrontal $\mathrm{rGBC} / \mathrm{fcMRI}$ in psychotic illness that may relate to time, severity, and co-morbidity. Such an approach, capitalizing on GBC's datadriven advantages and ability to deal with individual differences in connectivity patterns(36), may provide a tool for linking patterns of prefrontal dysconnectivity with psychotic illness heterogeneity.

\section{Limitations}

Present findings should be interpreted within the confines of several limitations. First, we allowed for co-morbid anxiety and history of drug/alcohol abuse/dependence to obtain a more ecologically-valid sample (although effects remain unchanged when we co-varied for these variables). Future studies should delineate to what extent present results replicate when examining subgroups with and without such co-morbid diagnoses. Second, patients were remitted ( 2 weeks) and we examined findings as a function of psychotic history. An important future direction is to examine the extent to which these patterns hold as severity of psychosis increases during mood episodes and to fully role out the possibility that differences in symptoms may reflect general psychopathology rather than psychosis history per se. Third, due to correlational nature of the analyses, it is unclear whether changes in connectivity reflect the cause of the mood disturbance versus the consequence of the illness. Thus, it will be critical to examine if connectivity patterns relate to illness duration, number of episodes and/or frequency of cycling, and manifest in at-risk populations. Fourth, despite convergence, the rGBC/seed-based findings are exploratory, given the voxel-wise search for prefrontal dysconnectivity and should also be verified using an independent replication. Similarly, it will be important to verify amgydala findings using identified mPFC and DLPFC as seeds via an independent sample (to ensure region selection independence(53)). This also applies to the individual difference analyses, which are not completely orthogonal to the originally presented results (thought they add convergent effects). Fifth, there is likely to be further functional specialization within the amygdala itself that we currently cannot capture in our study(65), which should be examined prospectively. Lastly, although when used as covariates medications did not alter the reported effects, reported patterns should be replicated in unmedicated samples(66).

\section{Conclusion}

Current findings substantially extend prior work in bipolar illness using a recently developed tool designed to detect global disruptions in prefrontal connectivity, applied to a wellpowered sample with carefully matched across-group demographics and SNR. We found reduced $\mathrm{mPFC}$ connectivity with the rest of PFC in bipolar disorder - a pattern that was inversely correlated with psychosis history. Critically, an independent amygdala seed-based 
analysis revealed elevated connectivity with a highly proximal $\mathrm{mPFC}$ region. These convergent yet independent effects highlight that $\mathrm{mPFC}$ dysconnectivity may represent a potential trait characteristic or risk factor of the disorder. Furthermore, given that the observed pattern of prefrontal dysconnectivity varied as a function of psychosis history (similar to findings in schizophrenia) suggests that disrupted PFC connectivity may be important for development of psychosis trans-diagnostically. Overall, our convergent findings suggest that disruption of prefrontal/limbic networks, particularly mPFC, may be a possible biomarker for bipolar disease risk.

\section{Supplementary Material}

Refer to Web version on PubMed Central for supplementary material.

\section{Acknowledgments}

Financial support for this study was provided by RO1 MH080912 (PI: DC Glahn). We would like to thank Dr. Pasko Rakic and Dr. Nenad Sestan for insightful comments and suggestions regarding current findings. We would also like to thank two anonymous Reviewers and Dr. Angus W. MacDonald III for their excellent feedback and constructive suggestions on how to improve this manuscript.

\section{References}

1. Vieta E, Phillips ML. Deconstructing bipolar disorder: a critical review of its diagnostic validity and a proposal for DSM-V and ICD-11. Schizophr Bull. 2007; 33:886-892. [PubMed: 17562693]

2. Phillips ML, Ladouceur CD, Drevets WC. A neural model of voluntary and automatic emotion regulation: implications for understanding the pathophysiology and neurodevelopment of bipolar disorder. Mol Psychiatry. 2008; 13:829, 833-857. [PubMed: 18574483]

3. Chen C-H, Suckling J, Lennox BR, Ooi C, Bullmore ET. A quantitative meta-analysis of fMRI studies in bipolar disorder. Bipolar Disord. 2011; 13:1-15. [PubMed: 21320248]

4. Altshuler L, Bookheimer S, Proenza MA, Townsend J, Sabb F, Firestine A, et al. Increased amygdala activation during mania: a functional magnetic resonance imaging study. Am J Psychiatry. 2005; 162:1211-1213. [PubMed: 15930074]

5. Blumberg HP, Kaufman J, Martin A, Whiteman R, Zhang JH, Gore JC, et al. Amygdala and hippocampal volumes in adolescents and adults with bipolar disorder. Arch Gen Psychiatry. 2003; 60:1201-1208. [PubMed: 14662552]

6. Chen CH, Suckling J, Lennox BR, Ooi C, Bullmore ET. A quantitative meta-analysis of fMRI studies in bipolar disorder. Bipolar Disord. 2011; 13:1-15. [PubMed: 21320248]

7. Strakowski SM, Delbello MP, Adler CM. The functional neuroanatomy of bipolar disorder: a review of neuroimaging findings. Mol Psychiatry. 2005; 10:105-116. [PubMed: 15340357]

8. Keener MT, Phillips ML. Neuroimaging in bipolar disorder: a critical review of current findings. Curr Psychiatry Rep. 2007; 9:512-520. [PubMed: 18221633]

9. Palaniyappan L, Cousins DA. Brain networks: foundations and futures in bipolar disorder. Journal of Mental Health. 2010; 19:157-167. [PubMed: 20433323]

10. Gray NA, Zhou R, Du J, Moore GJ, Manji HK. The use of mood stabilizers as plasticity enhancers in the treatment of neuropsychiatric disorders. The Journal of clinical psychiatry. 2003; 64:3-17. [PubMed: 12720479]

11. Schloesser RJ, Huang J, Klein PS, Manji HK. Cellular plasticity cascades in the pathophysiology and treatment of bipolar disorder. Neuropsychopharmacology. 2008; 33:110-133. [PubMed: 17912251]

12. Zarate CA, Singh J, Manji HK. Cellular plasticity cascades: targets for the development of novel therapeutics for bipolar disorder. Biol Psychiatry. 2006; 59:1006-1020. [PubMed: 16487491]

13. Raichle ME, Snyder AZ. A default mode of brain function: a brief history of an evolving idea. Neuroimage. 2007; 37:1083-1090. discussion 1097-1089. [PubMed: 17719799] 
14. Fox MD, Snyder AZ, Vincent JL, Corbetta M, Van Essen DC, Raichle ME. The human brain is intrinsically organized into dynamic, anticorrelated functional networks. Proc Natl Acad Sci USA. 2005; 102:9673-9678. [PubMed: 15976020]

15. Smith SM, Fox PT, Miller KL, Glahn DC, Fox PM, Mackay CE, et al. Correspondence of the brain's functional architecture during activation and rest. Proc Natl Acad Sci USA. 2009; 106:13040-13045. [PubMed: 19620724]

16. Vincent JL, Patel GH, Fox MD, Snyder AZ, Baker JT, Van Essen DC, et al. Intrinsic functional architecture in the anaesthetized monkey brain. Nature. 2007; 447:83-U84. [PubMed: 17476267]

17. Greicius MD, Supekar K, Menon V, Dougherty RF. Resting-state functional connectivity reflects structural connectivity in the default mode network. Cereb Cortex. 2009; 19:72-78. [PubMed: 18403396]

18. Fox MD, Greicius M. Clinical applications of resting state functional connectivity. Front Syst Neurosci. 2010; 4:19. [PubMed: 20592951]

19. Calhoun VD, Maciejewski PK, Pearlson GD, Kiehl KA. Temporal lobe and "default" hemodynamic brain modes discriminate between schizophrenia and bipolar disorder. Hum Brain Mapp. 2008; 29:1265-1275. [PubMed: 17894392]

20. Anand A, Li Y, Wang Y, Lowe MJ, Dzemidzic M. Resting state corticolimbic connectivity abnormalities in unmedicated bipolar disorder and unipolar depression. Psychiatry Res. 2009; 171:189-198. [PubMed: 19230623]

21. Chepenik LG, Raffo M, Hampson M, Lacadie C, Wang F, Jones MM, et al. Functional connectivity between ventral prefrontal cortex and amygdala at low frequency in the resting state in bipolar disorder. Psychiatry Res. 2010; 182:207-210. [PubMed: 20493671]

22. Price JL, Carmichael ST, Drevets WC. Networks related to the orbital and medial prefrontal cortex: a substrate for emotional behavior? Prog Brain Res. 1996; 107:523-536. [PubMed: 8782540]

23. Barbas H. Anatomic basis of cognitive-emotional interactions in the primate prefrontal cortex. Neuroscience and Behavioral Reviews. 1995; 19:499-510.

24. Barbas H. Flow of information for emotions through temporal and orbitofrontal pathways. J Anat. 2007; 211:237-249. [PubMed: 17635630]

25. Ghashghaei HT, Hilgetag CC, Barbas H. Sequence of information processing for emotions based on the anatomic dialogue between prefrontal cortex and amygdala. Neuroimage. 2007; 34:905923. [PubMed: 17126037]

26. Insel TR, Cuthbert BN. Endophenotypes: bridging genomic complexity and disorder heterogeneity. Biol Psychiatry. 2009; 66:988-989. [PubMed: 19900610]

27. Goodwin, FK.; Jamison, KR. Manic Depressive Illness. New York: Oxford University Press; 1990.

28. Dunayevich E, Keck PE Jr . Prevalence and Description of Psychotic Features in Bipolar Mania. Curr Psychiatry Rep. 2000; 2:286-290. [PubMed: 11122970]

29. Potash JB, Willour VL, Chiu YPD, Simpson SG, MacKinnon DF, Pearlson GD, et al. The Familial Aggregation of Psychotic Symptoms in Bipolar Disorder Pedigrees. Am J Psychiatry. 2001; 158:1258-1264. [PubMed: 11481160]

30. Bellivier F, Golmard JL, Henry C, Leboyer M, Schurhoff F. Admixture analysis of age at onset in bipolar I affective disorder. Arch Gen Psychiatry. 2001; 58:510-512. [PubMed: 11343533]

31. Coryell W, Leon AC, Turvey C, Akiskal HS, Mueller T, Endicott J. The significance of psychotic features in manic episodes: a report from the NIMH collaborative study. J Affect Disord. 2001; 67:79-88. [PubMed: 11869754]

32. Keck PE Jr, McElroy SL, Havens JR, Altshuler LL, Nolen WA, Frye MA, et al. Psychosis in bipolar disorder: phenomenology and impact on morbidity and course of illness. Compr Psychiatry. 2003; 44:263-269. [PubMed: 12923703]

33. Glahn DC, Bearden CE, Barguil M, Barrett J, Reichenberg A, Bowden CL, et al. The neurocognitive signature of psychotic bipolar disorder. Biol Psychiatry. 2007; 62:910-916. [PubMed: 17543288]

34. Strasser HC, Lilyestrom J, Ashby ER, Honeycutt NA, Schretlen DJ, Pulver AE, et al. Hippocampal and ventricular volumes in psychotic and nonpsychotic bipolar patients compared with 
schizophrenia patients and community control subjects: a pilot study. Biol Psychiatry. 2005; 57:633-639. [PubMed: 15780850]

35. Pearlson GD, Wong DF, Tune LE, Ross CA, Chase G, Links JM, et al. In vivo D2 dopamine receptor density in psychotic and nonpsychotic patients with bipolar disorder. Arch Gen Psychiatry. 1995; 52:471-477. [PubMed: 7771917]

36. Cole MW, Anticevic A, Repovs G, Barch DM. Variable global dysconnectivity and individual differences in schizophrenia. Biol Psychiatry. 2011; 70:43-50. [PubMed: 21496789]

37. Buckner RL, Sepulcre J, Talukdar T, Krienen FM, Liu H, Hedden T, et al. Cortical hubs revealed by intrinsic functional connectivity: mapping, assessment of stability, and relation to Alzheimer's disease. J Neurosci. 2009; 29:1860-1873. [PubMed: 19211893]

38. Cole MW, Pathak S, Schneider W. Identifying the brain's most globally connected regions. Neuroimage. 2010; 49:3132-3148. [PubMed: 19909818]

39. Martuzzi R, Ramani R, Qiu M, Shen X, Papademetris X, Constable RT. A whole-brain voxel based measure of intrinsic connectivity contrast reveals local changes in tissue connectivity with anesthetic without a priori assumptions on thresholds or regions of interest. Neuroimage. 2011; 58:1044-1050. [PubMed: 21763437]

40. First, MB.; Spitzer, RL.; Gibbon, M.; Williams, JBW. Structured clinical interview for the DSMIV-TR Axis I disorders. Washington, D. C: American Psychiatric Press; 2001.

41. Wechsler, D. Wechsler abbreviated scale of intelligence. San Antonio: The Psychological Corporation; 1999.

42. Glahn DC, Bearden CE, Cakir S, Barrett JA, Najt P, Serap Monkul E, et al. Differential working memory impairment in bipolar disorder and schizophrenia: effects of lifetime history of psychosis. Bipolar Disord. 2006; 8:117-123. [PubMed: 16542181]

43. Glahn DC, Bearden CE, Bowden CL, Soares JC. Reduced educational attainment in bipolar disorder. J Affect Disord. 2006; 92:309-312. [PubMed: 16524631]

44. Hamilton M. Development of a rating scale for primary depressive illness. Br J Soc Clin Psychol. 1967; 6:278-296. [PubMed: 6080235]

45. Young R, Biggs J, Ziegler V, Meyer D. A rating scale for mania: reliability, validity and sensitivity. Br J Psychiatry. 1978; 133:429-435. [PubMed: 728692]

46. Ventura J, Green MF, Shaner A, Liberman RP. Training and quality assurance with the Brief Psychiatric Rating Scale: "The drift busters". International Journal of Methods in Psychiatric Research. 1993; 3:221-244.

47. Fischl B, Salat DH, Busa E, Albert M, Dieterich M. Whole Brain Segmentation Automated Labeling of Neuroanatomical Structures in the Human Brain. Neuron. 2002; 33:341-355. [PubMed: 11832223]

48. Anticevic A, Repovs G, Barch DM. Resisting Emotional Interference: Brain Regions Facilitating Working Memory Performance During Negative Distraction. Cogn Affect Behav Neurosci. 2010; 10:159-173. [PubMed: 20498341]

49. Anticevic A, Repovs G, Barch DM. Emotion Effects on Attention, Amygdala Activation, and Functional Connectivity in Schizophrenia. Schizophr Bull. 2011 [Epub ahead of print].

50. Anticevic A, Repovs G, Shulman GL, Barch DM. When less is more: TPJ and default network deactivation during encoding predicts working memory performance. Neuroimage. 2010; 49:2638-2648. [PubMed: 19913622]

51. Fischl B, Salat DH, van der Kouwe AJ, Makris N, Ségonne F, Quinn BT, et al. Sequenceindependent segmentation of magnetic resonance images. Neuroimage. 2004; 23(Suppl 1):S69-84. [PubMed: 15501102]

52. Forman SD, Cohen JD, Fitzgerald M, Eddy WF, Mintun MA, Noll DC. Improved assessment of significant activation in functional magnetic resonance imaging (fMRI): Use of a cluster-size threshold. Magnetic Resonance Medicine. 1995; 33:636-647.

53. Kriegeskorte N, Simmons WK, Bellgowan PSF, Baker CI. Circular analysis in systems neuroscience: the dangers of double dipping. Nat Neurosci. 2009; 12:535-540. [PubMed: 19396166] 
54. Chai XJ, Whitfield-Gabrieli S, Shinn AK, Gabrieli JD, Nieto Castanon A, McCarthy JM, et al. Abnormal Medial Prefrontal Cortex Resting-State Connectivity in Bipolar Disorder and Schizophrenia. Neuropsychopharmacology. 2011; 36:2009-2017. [PubMed: 21654735]

55. Beckmann M, Johansen-Berg H, Rushworth MF. Connectivity-based parcellation of human cingulate cortex and its relation to functional specialization. J Neurosci. 2009; 29:1175-1190. [PubMed: 19176826]

56. Hutchison RM, Womelsdorf T, Gati JS, Leung LS, Menon RS, Everling S. Resting-state connectivity identifies distinct functional networks in macaque cingulate cortex. Cereb Cortex. 2011; 22:1294-1308. [PubMed: 21840845]

57. Johansen-Berg H, Gutman DA, Behrens TE, Matthews PM, Rushworth MF, Katz E, et al. Anatomical connectivity of the subgenual cingulate region targeted with deep brain stimulation for treatment-resistant depression. Cereb Cortex. 2007; 18:1374-1383. [PubMed: 17928332]

58. Price JL, Drevets WC. Neural circuits underlying the pathophysiology of mood disorders. Trends in Cognitive Sciences. 2011; 16:61-71. [PubMed: 22197477]

59. Lewis CM, Baldassarre A, Committeri G, Romani GL, Corbetta M. Learning sculpts the spontaneous activity of the resting human brain. Proc Natl Acad Sci U S A. 2009; 106:1755817563. [PubMed: 19805061]

60. Quirk GJ, Garcia R, González-Lima F. Prefrontal mechanisms in extinction of conditioned fear. Biol Psychiatry. 2006; 60:337-343. [PubMed: 16712801]

61. Kang HJ, Kawasawa YI, Cheng F, Zhu Y, Xu X, Li M, et al. Spatio-temporal transcriptome of the human brain. Nature. 2011; 478:483-489. [PubMed: 22031440]

62. Keshavan MS, Morris DW, Sweeney JA, Pearlson GD, Thaker GK, Seidman LJ, et al. A dimensional approach to the psychosis spectrum between bipolar disorder and schizophrenia: the Schizo-Bipolar Scale. Schizophr Res. 2011; 133:250-254. [PubMed: 21996268]

63. Meda SA, Gill A, Stevens MC, Lorenzoni RP, Glahn DC, Calhoun VD, et al. Differences in Resting-State Functional Magnetic Resonance Imaging Functional Network Connectivity Between Schizophrenia and Psychotic Bipolar Probands and Their Unaffected First-Degree Relatives. Biol Psychiatry. 2012 [Epub ahead of print].

64. Soyka M, Koch W, Möller HJ, Rüther T, Tatsch K. Hypermetabolic pattern in frontal cortex and other brain regions in unmedicated schizophrenia patients. Results from a FDG-PET study. Eur Arch Psychiatry Clin Neurosci. 2005; 255:308-312. [PubMed: 15834758]

65. Laviolette SR, WJL, Grace AA. A subpopulation of neurons in the medial prefrontal cortex encodes emotional learning with burst and frequency codes through a dopamine D4 receptordependent basolateral amygdala input. J Neurosci. 2005; 25:6066-6075. [PubMed: 15987936]

66. Phillips ML, Travis MJ, Fagiolini A, Kupfer DJ. Medication effects in neuroimaging studies of bipolar disorder. Am J Psychiatry. 2008; 165:313-320. [PubMed: 18245175]

67. Levinson DF, Mowry BJ, Escamilla MA, Faraone SV. The Lifetime Dimensions of Psychosis Scale (LDPS): description and interrater reliability. Schizophr Bull. 2002; 28:683-695. [PubMed: 12795498] 


\section{Prefrontal rGBC in Bipolar I Disorder}

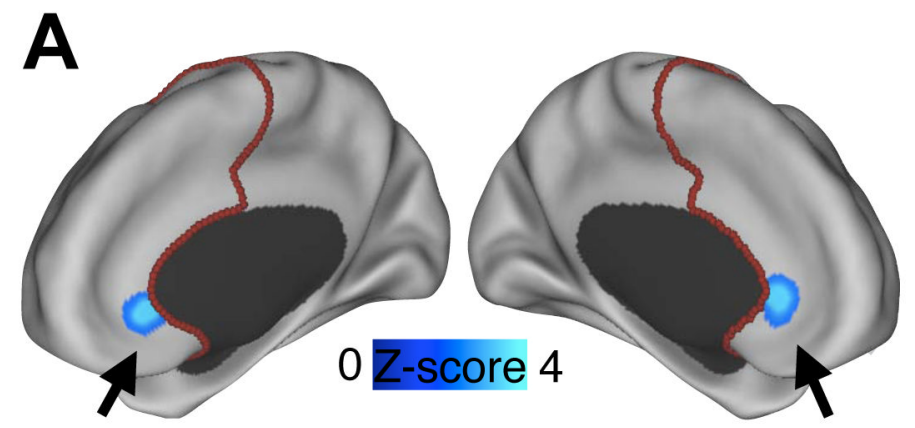

B

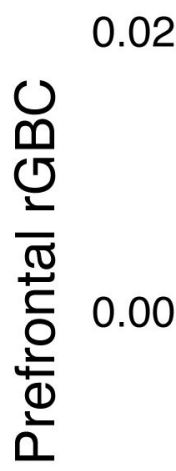

- Controls

- BPW

-BPP

Figure 1. Global Prefrontal Dysconnectivity

(a) Significant between-group differences in prefrontal rGBC between bipolar patients and healthy participants revealed a medial prefrontal cortex region (MPFC) $(x=3, y=32, z=1)$. The red border approximately marks the restricted PFC analysis. (b) rGBC values are shown for the $\mathrm{mPFC}$ region across the three groups; healthy participants (white) and bipolar patients without psychosis history (BPW, gray); bipolar patients with history of psychosis (BPP, black). Error bars represent $+/-1$ standard error of the mean. 
Amygdala-Prefrontal fcMRI in Bipolar I Disorder
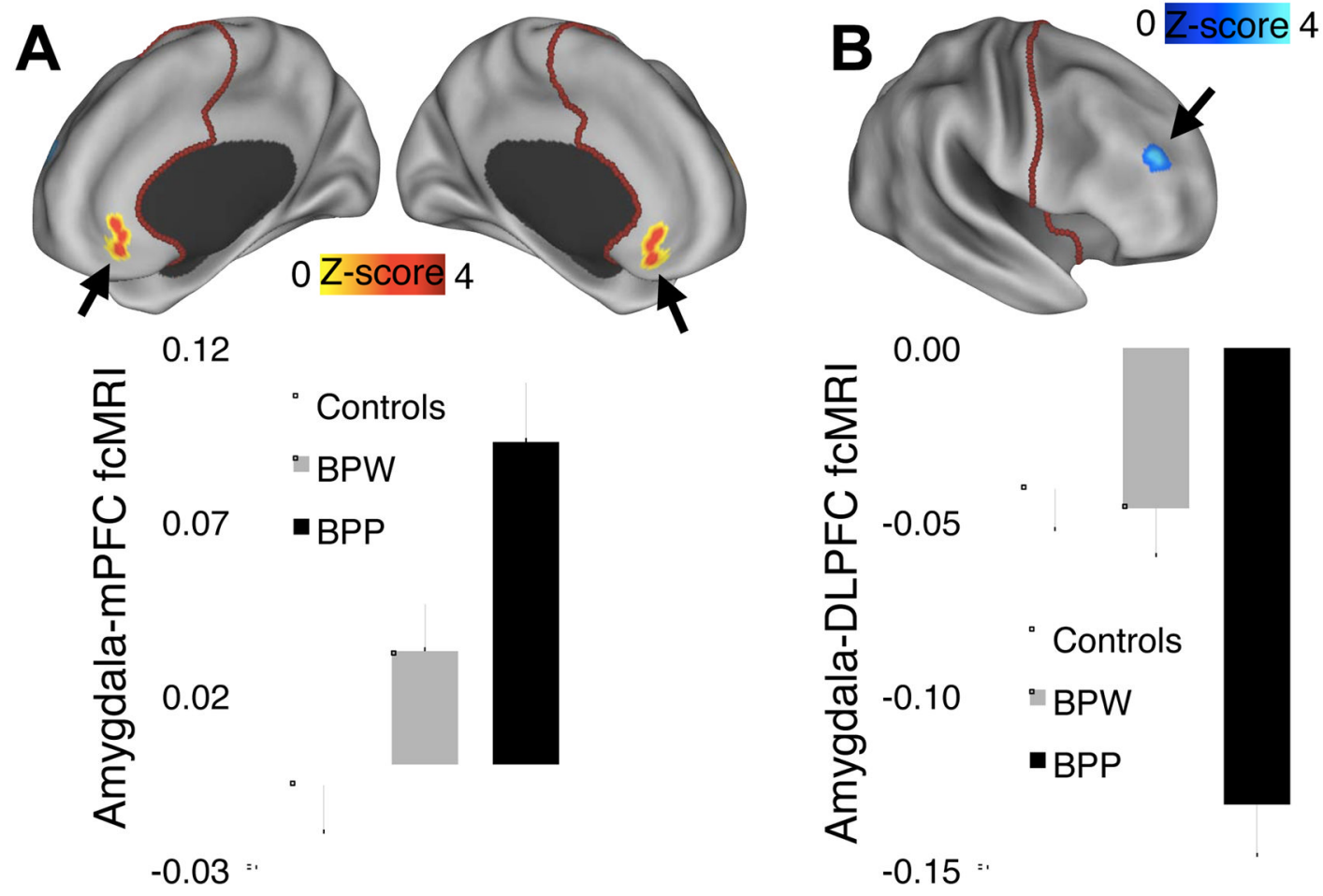

Figure 2. Amygdala Prefrontal Dysconnectivity

Significant group differences in amygdala-prefrontal fcMRI between bipolar disorder subgroups and healthy controls. (a) Yellow/red foci mark regions where bipolar patients with a history of psychosis showed increased amygdala connectivity relative to nonpsychotic patients and healthy controls. This pattern was centered on the medial prefrontal cortex (mPFC) $(x=1, y=41, z=-3)$. The red border approximately marks the restricted PFC analysis. (b) A right DLPFC region ( $\mathrm{x}=34, \mathrm{y}=43, \mathrm{z}=30$ ) is shown in blue for which bipolar patients with a history of psychosis showed decreased amygdala connectivity relative to non-psychotic patients and healthy controls. The rGBC values are shown across both foci for controls (white), bipolar patients without a history of psychosis (BPW, grey) and bipolar patients with history of psychosis (BPP, black). Error bars represent $+/-1$ standard error of the mean. 


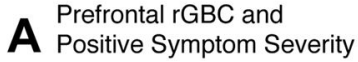

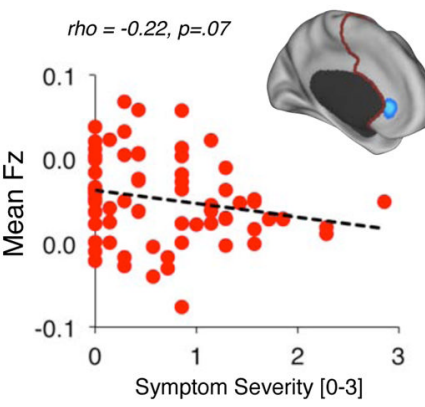

Amygdala-mPFC fcMRI and

Positive Symptom Severity

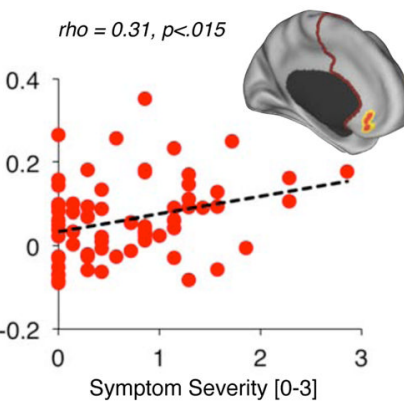

Amygdala-DLPFC fcMRI and

Positive Symptom Severity

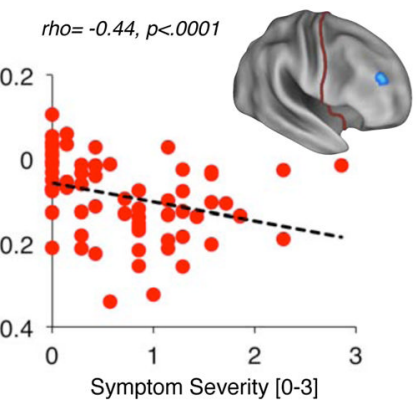

Figure 3. rGBC, Amygdala-Prefrontal Dysconnectivity and Lifetime Psychotic Symptom Severity

(a) Trend-level inverse relationship between $\mathrm{mPFC}$ rGBC and lifetime positive psychotic symptom severity across the entire sample of bipolar patients $(\mathrm{rho}=-.22, \mathrm{p}=.07)$. (b) Significant positive relationship between amygdala-mPFC fcMRI and lifetime positive psychotic symptom severity across the entire sample of bipolar patients (rho=.31, $\mathrm{p}<.015)$. (c) Significant inverse relationship between amygdala-DLPFC fcMRI and lifetime positive psychotic symptom severity across the entire sample of bipolar patients (rho= $=.44, \mathrm{p}<$. 0001). Direction of all reported individual difference effects show strong convergence with main effects. The scale on the $\mathrm{x}$-axis captures a clinician-rated severity index that ranges from 0 (absent) to 4 (Very severe; Gross or nearly constant effect on function) (67). 


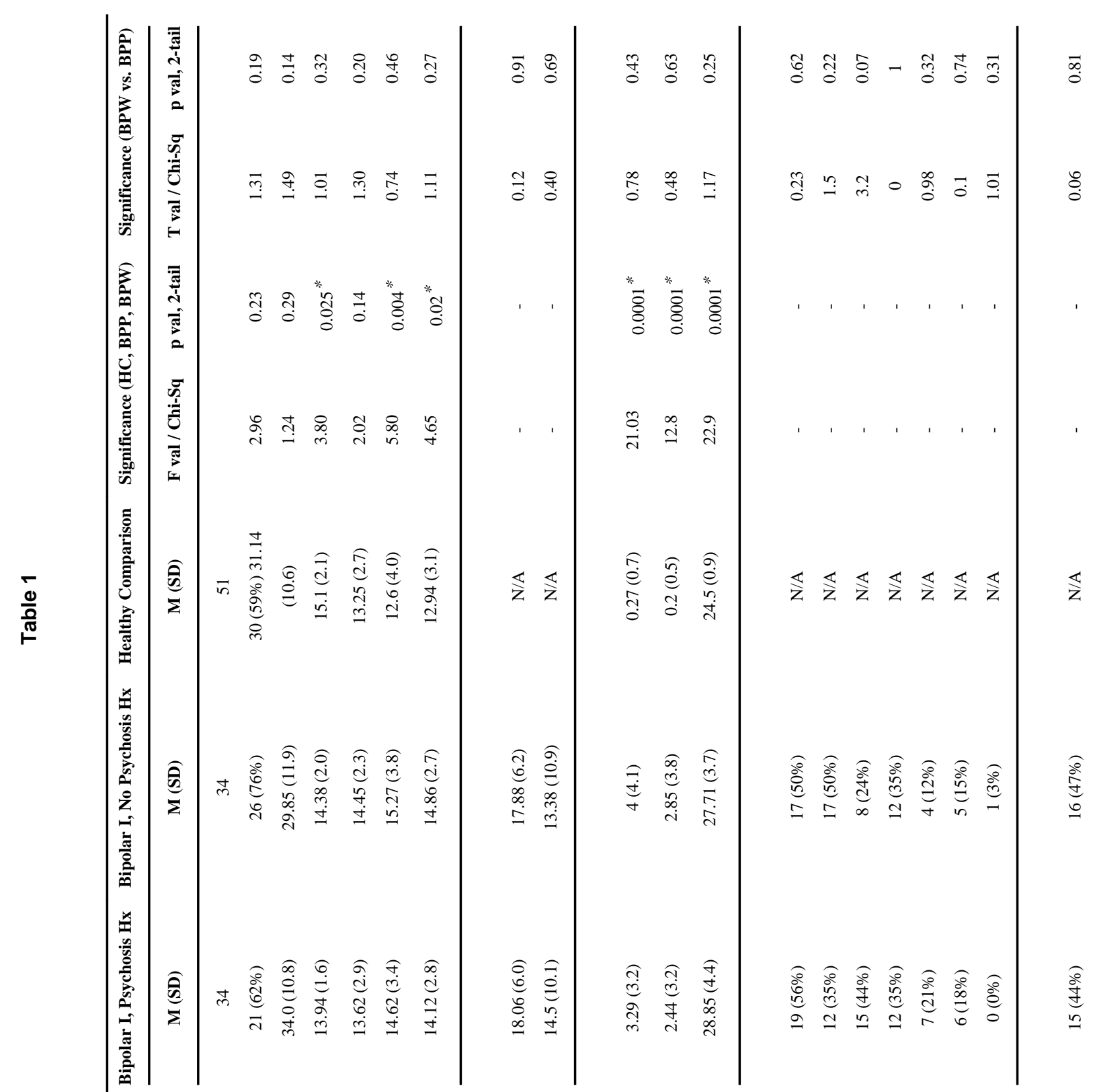

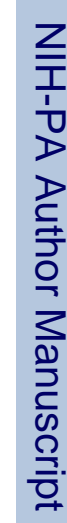

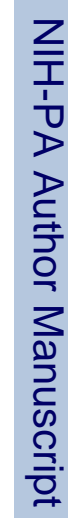

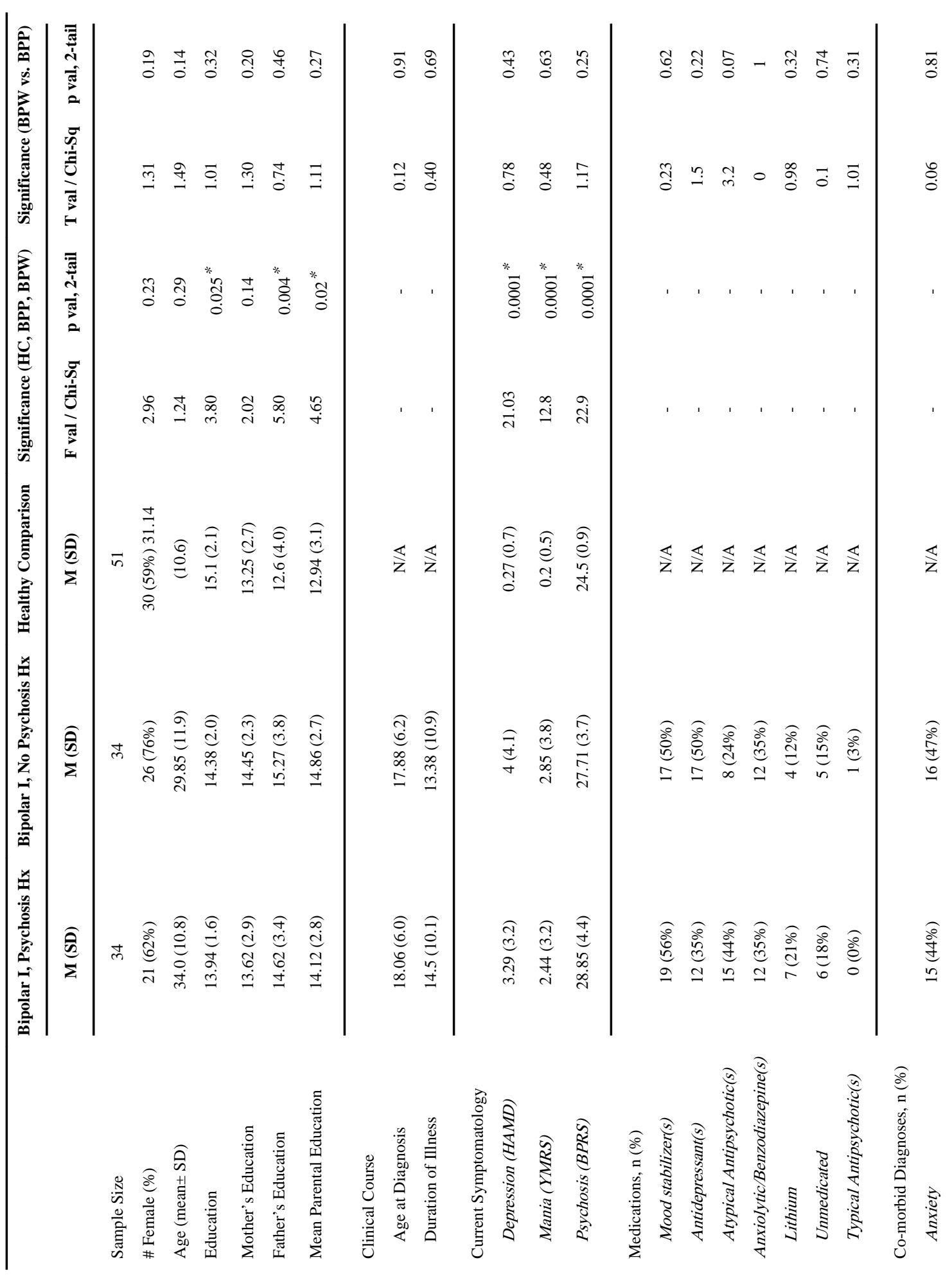
Biol Psychiatry. Author manuscript; available in PMC 2014 March 15. 


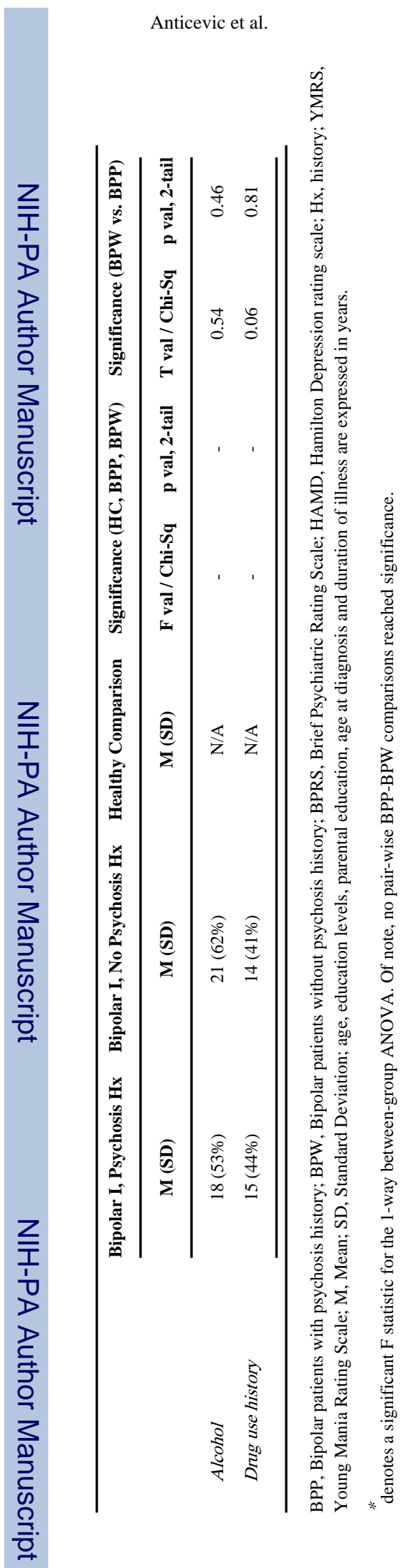

Biol Psychiatry. Author manuscript; available in PMC 2014 March 15. 


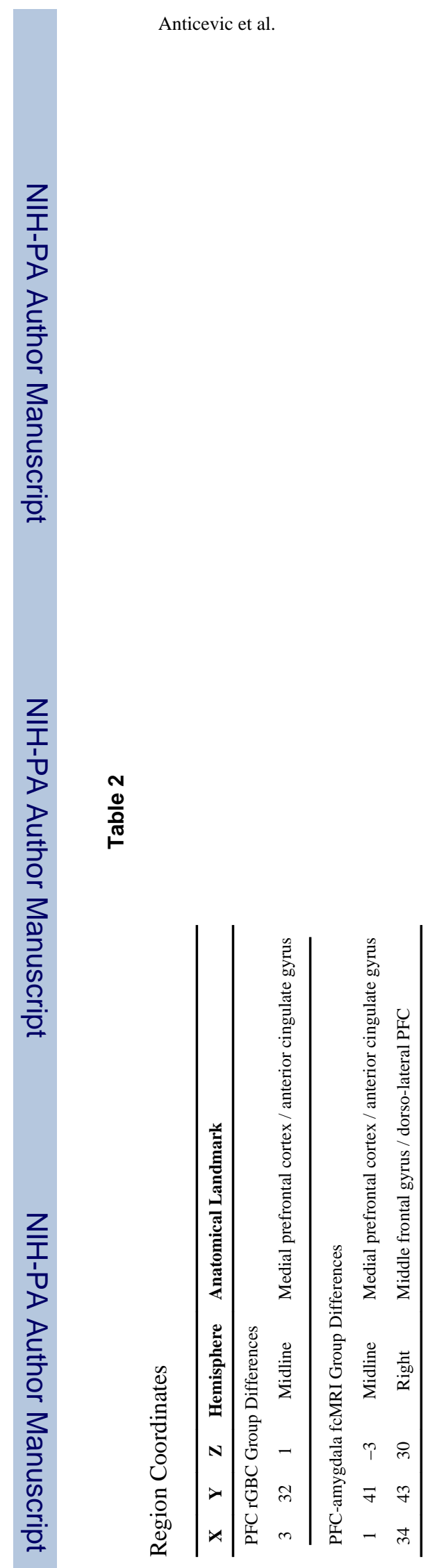

Biol Psychiatry. Author manuscript; available in PMC 2014 March 15. 